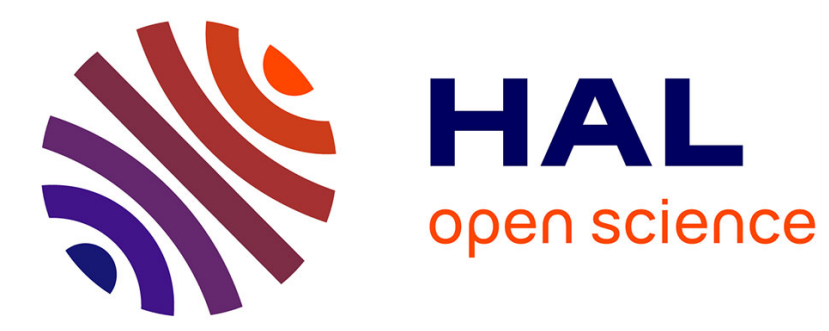

\title{
Une nouvelle pierre gravée dans la grotte du Chaffaud (Savigné, Vienne) \\ Pascaline Gaussein
}

\section{To cite this version:}

Pascaline Gaussein. Une nouvelle pierre gravée dans la grotte du Chaffaud (Savigné, Vienne). Bulletin de la Société préhistorique française, 2014, 111 (1), pp.139-142. 10.3406/bspf.2014.14371 . hal01529108

\section{HAL Id: hal-01529108 \\ https://hal.parisnanterre.fr/hal-01529108}

Submitted on 30 May 2017

HAL is a multi-disciplinary open access archive for the deposit and dissemination of scientific research documents, whether they are published or not. The documents may come from teaching and research institutions in France or abroad, or from public or private research centers.
L'archive ouverte pluridisciplinaire HAL, est destinée au dépôt et à la diffusion de documents scientifiques de niveau recherche, publiés ou non, émanant des établissements d'enseignement et de recherche français ou étrangers, des laboratoires publics ou privés. 


\title{
Une nouvelle pierre gravée dans la grotte du Chaffaud (Savigné, Vienne)
}

\author{
Pascaline GAUSSEIN
}

L A GROTTE du Puits au Chaffaud (dite grotte du Chaffaud) se trouve sur les rives de la Charente, sur la commune de Savigné (Vienne). Elle a été fouillée pour la première fois dans les années 1830, puis jusqu'en 1866 par différents érudits et chercheurs. Entre 1867 et 1984, le site a été abandonné aux visiteurs et aux amateurs. La reprise des déblais et de sédiments bréchifiés en 1985 et 1986 a permis de compléter l'approche de la stratigraphie complexe du site (Airvaux, 2002; Gaussein, 2012). De nouveaux vestiges ont ressurgi des déblais et des greniers. C'est le cas de la pierre gravée inédite qui fait l'objet de cet article. Nous faisons ici une synthèse de l'analyse de cette pièce, menée dans le cadre d'un master de recherche (Gaussein, 2012). Nous proposons de replacer cette pierre gravée dans son contexte géographique et technoculturel, et de mettre en perspective ces nouvelles données.

\section{Nouvelles données}

\section{Objet trouvé, (Pré) histoire perdue}

La pierre gravée inédite a été découverte en 1969 par Patrice Kieffer, amateur, au cours d'une visite du Chaffaud. Dans un courrier, l'inventeur indique le lieu de la découverte sur une carte manuscrite : un petit diverticule près de l'entrée est. Il précise que le petit bloc gravé a été trouvé dans un « tas de cailloux bouleversés », avec des silex et os « taillés ». Il s'agit donc très certainement d'un tas de déblais de fouilles ou de pillages anciens. Longtemps considérée par son inventeur comme un polissoir, cette pièce n'a été authentifiée qu'en 2008 par Oscar Fuentes, directeur du centre d'interprétation du Rocaux-Sorciers (Vienne). Elle a ensuite été prise en charge octobre 2010 par le service régional de l'Archéologie du Poitou-Charentes.

\section{Étude de la pièce}

Pour le relevé des tracés et la compilation des différentes informations liées aux états de surface de la pièce, nous avons utilisé la technique du calque sur photographie.

Le support sub-rectangulaire mesure $13 \mathrm{~cm}$ de longueur pour $9,5 \mathrm{~cm}$ de largeur, et son épaisseur maximale est de 4,5 cm. Il s'agit d'un fragment de plancher stalagmitique de patine orangée (fig. 1). Des coups (de pioche?) ont laissé des écrasements blancs sur la pierre. Les surfaces du support sont relativement planes. La face gravée est légèrement ondulée et fortement dégradée sur certaines zones et sur ses bords. Sur les tranches

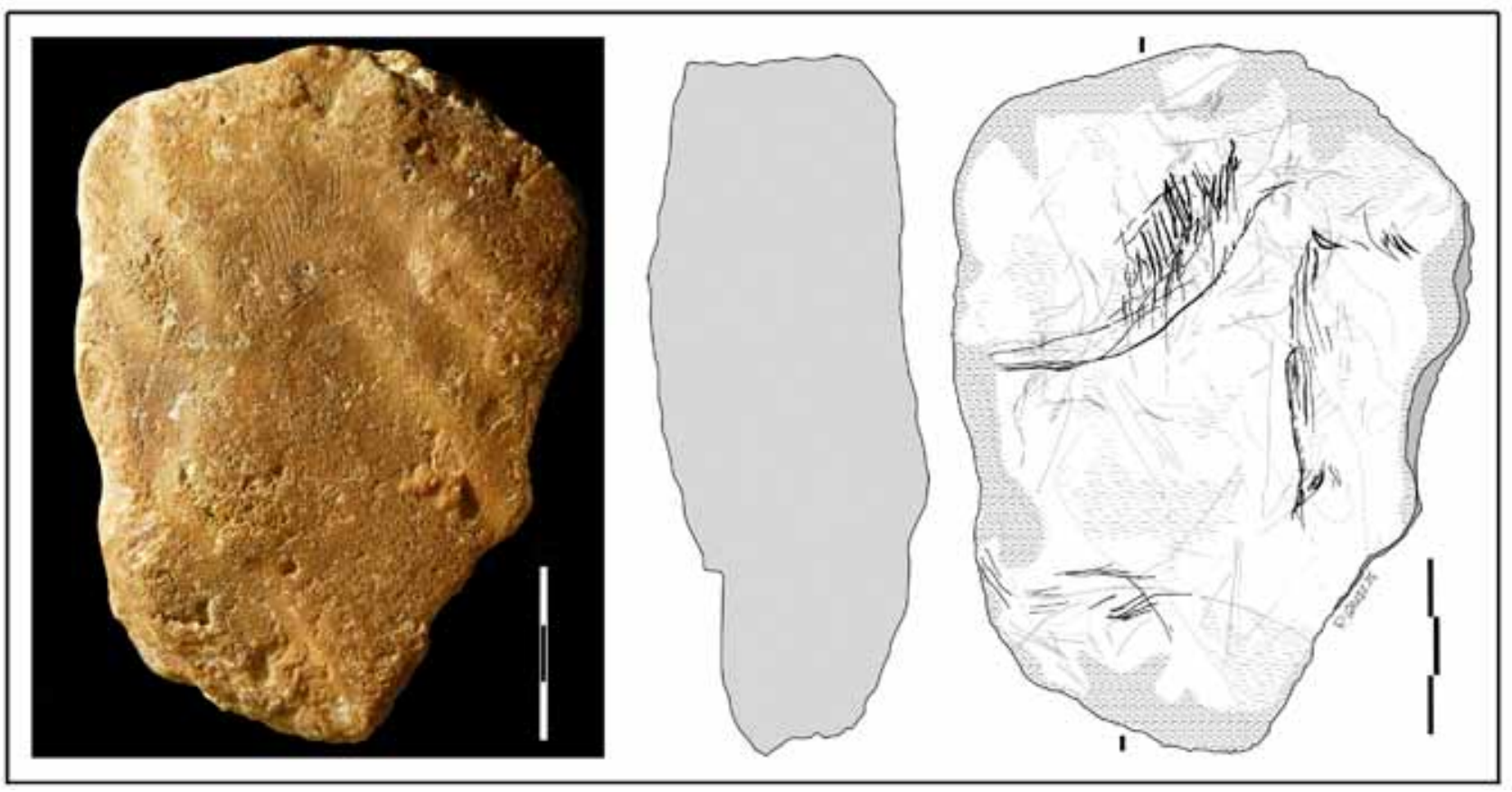

Fig. 1 - Fragment de plancher stalagmitique gravé d'une figure de cheval, le Puits au Chaffaud (Savigné, Vienne). Ligne de tirets : reliefs; plage de tirets : altération de la surface (cliché, relevé et DAO P. Gaussein). 
irrégulières du bloc, la structure en longs cristaux est caractéristique des formations stalagmitiques. Le bord gauche présente une zone fracturée qui interrompt les incisions du recto.

L'hypothèse d'une œuvre relevant de l'art pariétal, issue d'une paroi fragmentée (effondrements de voûte, fouilles du XIX ${ }^{\mathrm{e}}$ siècle), n'est pas envisagée (comm. écrite de J. Airvaux). Des blocs de calcite ont été relevés dans le niveau non remanié (couche VI : Airvaux, 2002), et les motifs et figures des autres blocs gravés du site sont globalement complets ou seulement tronqués en un coin. Il sagirait donc bien de vestiges d'un art mobilier.

Seule une face est gravée. Elle porte des tracés simples de largeur et de profondeur moyenne (inférieures à $1 \mathrm{~mm}$ ), dont le fond est de section principalement en U. Des tracés plus fins (inférieurs à $0,5 \mathrm{~mm}$ ) présentent un fond de section en $\mathrm{V}$.

Nous avons identifié l'avant-train d'un cheval représenté en profil droit (fig. 1). Deux séquences juxtaposées de tracés rectilignes parallèles évoquent une crinière en brosse, caractéristique des représentations de chevaux paléolithiques. Le corps est massif. Il est délimité par les tracés multiples d'un ventre rond et lourd et d'un dos rectiligne. Une ligne de dos profondément incisée est prolongée et dessine une encolure trop fine. Quelques tracés plus fins et irréguliers rejoignent la crinière et esquissent une encolure aux proportions plus naturelles. La crinière a pu être complétée d'un tracé continu supérieur, mais il est très altéré voire douteux. Quelques tracés de tête sont visibles : l'extrémité d'une barbe, et un tracé profondément gravé que nous attribuons avec réserve à la joue du cheval. De longs tracés illustrent le pelage du cou et la bosse du poitrail : il s'agit probablement de la représentation d'un cheval en période hivernale (Crémades, 1997).

Ces zones lacunaires (tête et membres) ne semblent pas avoir été affectées par les détériorations observées par ailleurs sur le bloc. L'analyse microtopographique ne révèle aucun vestige de tracé. Ce cheval semblerait donc inachevé. Les tracés de dos et de ventre sont interrompus par le bord gauche dégradé du bloc, et inclinés au niveau du pli inguinal de l'animal. Ce support a pu être plus grand, orné d'un cheval en pied.

\section{Technologie de la pierre gravée}

Nous avons également effectué des analyses au microrugosimètre (station de micromesures confocales STILCHR 150 au Centre de recherche et de restauration des musées de France). Cette technique de microtopographie permet d'opérer des mesures et observations technologiques à haute résolution d'une surface en trois dimensions et une meilleure lecture des surfaces et des tracés (Mélard, 2008).

Les différentes étapes de réalisation de la crinière ont pu être précisées. La séquence de tracés sub-verticaux semble avoir été incisée en premier lieu, suivie de la séquence de tracés obliques. Le probable tracé longitudinal paraît lui aussi postérieur aux traits verticaux. À l'extrémité distale des tracés verticaux de la crinière, de petites cupules d'écrasement de la matière pourraient indiquer l'entame du trait, le point d'impact où la partie active de l'outil rencontre la matière avant de l'inciser (fig. 2a). Le sens d'incision du tracé est ainsi observable : du haut vers le bas.

Après avoir croisé les différentes données enregistrées, voici un condensé des observations technologiques effectuées. La largeur et la profondeur des tracés ne sont pas systématiquement corrélées. La différence entre les gestes exécutés pour inciser la ligne de dos et la séquence de traits de la crinière pourrait expliquer certaines nuances de mesures enregistrées. En effet, la ligne de dos est constituée d'un long tracé : il est donc possible d'exercer plus de force sur l'outil, contrairement aux tracés plus courts de la crinière. Nous avons également pu constater que les différents profils de traits gravés ne répondaient pas nécessairement à un ordre de mesures spécifique. La figure $2 b$ illustre cette variabilité des mesures et des sections au cours d'une même incision. Cette variabilité peut être due à des différences de dureté des grains et d'altération de la matière, mais aussi à la dégradation progressive de la partie active de l'outil. L'usure, le ravivage de la partie active ou encore le changement d'outil de gravure sont également des paramètres à prendre en compte pour expliquer des variations de profil des tracés. Il semble donc difficile d'identifier l'outil utilisé.

\section{Hypothèses d'attribution au Magdalénien}

\section{En faveur du Magdalénien moyen}

La représentation de la crinière en séquences de tracés parallèles obliques rappelle les crinières en brosse du Magdalénien moyen de la Vienne. L'importance accordée au pelage de l'animal permet également de rattacher cette figure à la tendance naturaliste et réaliste des graveurs du Magdalénien moyen de la région, tendance observée à la Marche et au réseau Guy-Martin à Lussac-les-Châteaux, et au Taillis-des-Coteaux à Antigny (Gaussein, 2012). La facture peu soignée du dessin ne va pas à l'encontre de ces pistes chronostylistiques, puisqu'une forte variabilité de la qualité du dessin a été observée au sein de chaque site de la région.

Il y a peu, nous aurions rapproché cette pierre du " faciès de type Lussac-Angles », caractérisé par ses représentations figuratives naturalistes et réalistes. Mais sa distinction du "faciès de la Garenne » commence à être remise en question, notamment depuis la découverte de figurations animalières relativement réalistes dans le site éponyme (Paillet, 2009). Qui plus est, navettes et sagaies de type Lussac-Angles ont été mises au jour au Chaffaud (Airvaux, 2002). Nous ne proposerons donc pas une attribution plus précise en faveur de l'un ou l'autre des faciès du Magdalénien moyen.

\section{En faveur d'un Magdalénien plus récent}

La délimitation de la crinière par une ligne continue serait plutôt caractéristique de faciès plus récents. Les cri- 
nières ainsi figurées sont généralement retrouvées dans les collections attribuées au Magdalénien supérieur et final, comme c'est le cas pour trois autres chevaux figurés sur des pierres du Chaffaud (Airvaux, 2002; Airvaux et Leuvrey, 2009). Mais nous insistons sur le fait que, pour ce nouveau cheval du Chaffaud, son existence demeure très douteuse. Le pelage, la bosse du poitrail et le ventre lourd rappellent également les chevaux du même site. Ces détails sont de taille, puisqu'ils permettent également de comparer ces figures aux chevaux du Magdalénien supérieur de Dordogne. Nous relevons des similitudes particulièrement intéressantes avec les gravures sur pierre de Limeuil, de la Madeleine, et celles sur les dalles de la grotte de la Mairie à Teyjat. L'allure figée, la tête haute, les formes et le pelage permettent de rapprocher la figure inédite de certains chevaux pariétaux de la grotte Margot en Mayenne, également attribuée à un Magdalénien récent.

\section{Sur la route du silex}

La cohérence de nos rapprochements avec des régions voisines et plus lointaines repose notamment sur la circulation de matières premières siliceuses, qui traduit des
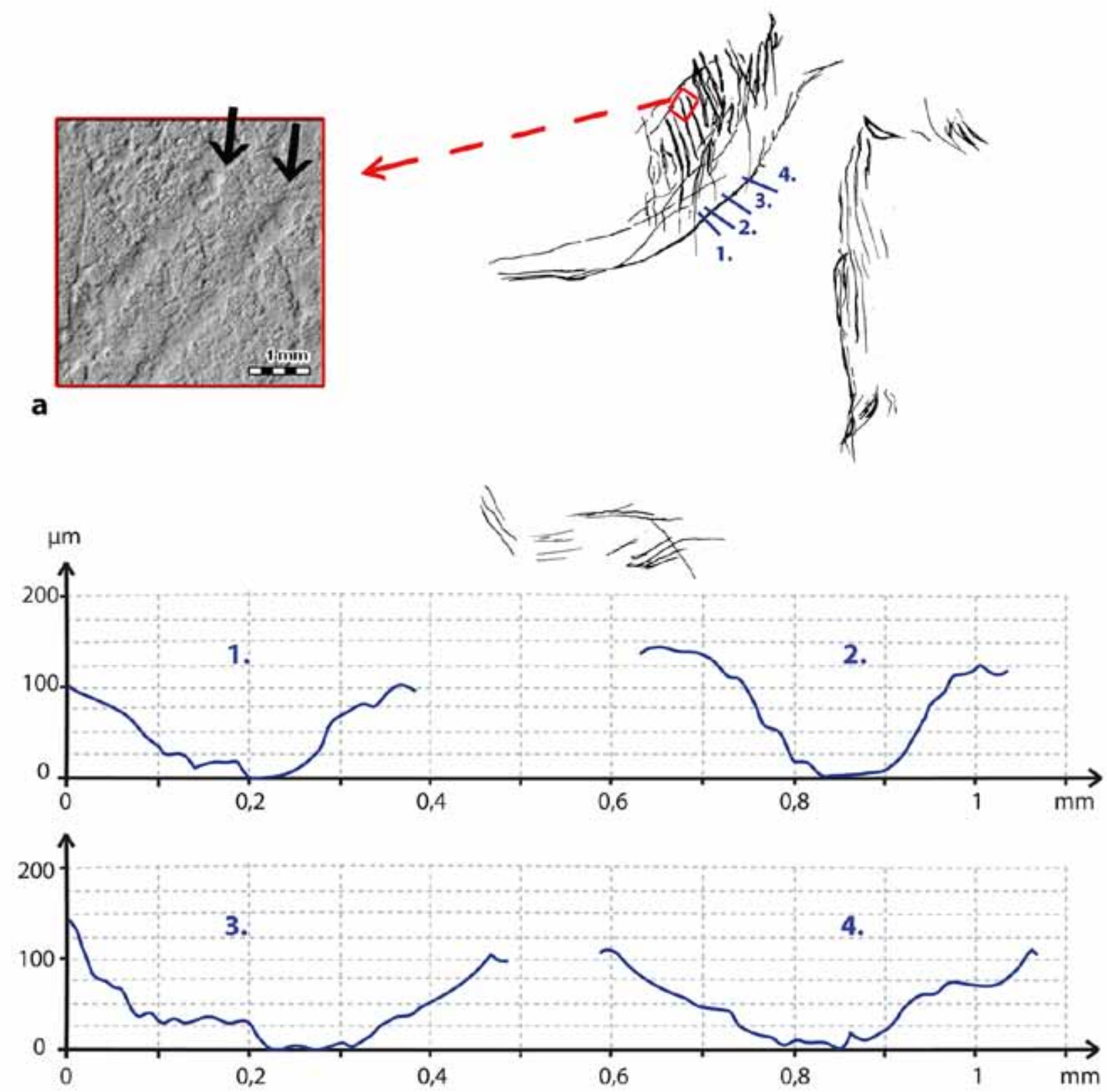

b

Fig. 2 - Analyse microtopographique de la nouvelle figure gravée du Chaffaud (Savigné, Vienne). a : simulation photoréaliste avec lumière rasante artificielle; détail de l'entame de deux tracés de crinière. $\mathrm{b}$ : sections successives du tracé principal de la ligne de dos du cheval (logiciel Mountain Map Universal). 
échanges directs ou de proche en proche entre chaque microculture magdalénienne. Les grottes du Chaffaud se situent sur une voie de passage et d'échange entre Bassin parisien et Bassin aquitain, et plus spécifiquement entre la région du Grand-Pressigny au nord et la région du Bergeracois (Dordogne) au sud. Or, les matériaux de Touraine (silex du Grand-Pressigny, jaspes de Fontmaure) sont retrouvés tant vers le sud, jusque dans le Tarn-etGaronne (Primault, 2003; Langlais, 2010) que vers le nord, dans la vallée de l'Erve en Mayenne (comm. orale J. Primault, 2013). Réciproquement, du silex bergeracois a été identifié dans des sites de la Vienne, au Roc-auxSorciers à Angles-sur-l'Anglin (comm. orale J. Primault, 2013). La proximité stylistique des figures du Chaffaud et des chevaux de la Dordogne est donc cohérente sur les plans techno-économique (circulation du silex) et géographique (bassin versant de la Charente).

\section{Conclusions}

Cette nouvelle gravure sur pierre s'inscrit bien dans le contexte culturel connu dans la Vienne au Magdalénien. Cette pièce nous rappelle cependant les limites de l'approche chronostylistique des représentations figuratives. Cette étude ouvre des pistes de réflexion autour du territoire et de l'organisation sociale des groupes magdaléniens. Derrière un certain réalisme, le manque de conformisme du dessin nous mène à penser que dans la Vienne, au Magdalénien (moyen et/ou supérieur), la gravure de la pierre n'était peut-être pas réservée à quelques initiés, détenteurs de savoirs cosmologiques, rituels et artistiques (Tosello, 2003; Gaussein, 2012).

Remerciements : Nous remercions nos directeur et tuteur de mémoire D. Vialou et P. Paillet, ainsi que J. Primault et J.-J. Ezrati pour leurs conseils. À l'inventeur de cette pierre, P. Kieffer, nous devons la reconnaissance de sa curiosité d'amateur et de son honnêteté scientifique.

\section{RÉFÉRENCES BIBLIOGRAPHIQUES}

Airvaux J. (2002) - Le Chaffaud, 168 ans après : à la mémoire de notre ami Henri Reigner qui participa aux recherches, Préhistoire du Sud-Ouest, 9, 1, p. 1-52.

Airvaux J., Leuvrey J.-M. (2009) - Nouvelle découverte d'une œuvre de gravure à la grotte du Puits au Chaffaud à Savigné, Vienne, Préhistoire du Sud-Ouest, 17, 1, p. 115-125.

CRÉMADEs M. (1997) - Bestiaire figuré, environnement animal, saisonnalité à la grotte de la Vache (Alliat, Ariège), Bulletin de la Société préhistorique française, 94, 4, p. 455-470.

GAUSSEIN P. (2012) - Art gravé sur supports mobiliers lithiques de la Vienne magdalénienne. Analyses stylistique, technologique et tracéologique : la figure du cheval, mémoire de master 2, Muséum national d'histoire naturelle, Paris, 210 p.

LANGlais M. (2010) - Les Sociétés magdaléniennes de l'isthme pyrénéen, Paris, CTHS, $336 \mathrm{p}$.

MÉLARD N. (2008) - Les pierres gravées de la Marche à Lussacles-Châteaux (Vienne). Techniques, technologie et interprétations, Gallia Préhistoire, 50, p. 143-270.

PAillet P. (2009) - L'art mobilier sur supports lithiques de « la Garenne » (Saint-Marcel, Indre), in J. Despriée, S. Tymula et A. Rigaud (dir), Données récentes sur le Magdalénien de «la Garenne » (Saint-Marcel, Indre). La place du Magdalénien " à navettes » en Europe, Argenton-sur-Creuse, ASSAM (Numéro spécial du Bulletin de l'Association pour la sauvegarde du site archéologique d'Argentomagus et amis du musée, 2), p. 181-200.

Primault J. (2003) - Exploitation et diffusion des silex de la région du Grand-Pressigny au Paléolithique, thèse de doctorat, université Paris X, Nanterre, 362 p.

Tosello G. (2003) - Pierres gravées du Périgord magdalénien, art, symboles, territoires, Paris, CNRS (Supplément à Gallia Préhistoire, 36), 577 p.

Pascaline Gaussein

Doctorante à l'université Paris-Ouest, Nanterre UMR 7055 « Préhistoire et technologie » 1 rue de Montreuil 75011 Paris pgaussein@hotmail.fr 